\title{
Simulated performance of an energy storage and return prosthetic ankle based on cams and miniature hydraulics
}

\author{
Anna Pace, Member, IEEE, James Gardiner, Laurence Kenney, David Howard
}

\begin{abstract}
Prosthetic feet are limited in their ability to mimic the energy-recycling behaviour of an intact ankle, negatively affecting lower-limb amputees' gait in terms of metabolic cost and walking speed. To overcome these weaknesses, a novel prosthetic ankle based on hydraulics is described here. The ankle joint drives two cams, which in turn drive two hydraulic rams. One cam-ram system captures the negative work done from foot-flat until maximum dorsiflexion, by pumping oil into an accumulator, while the other returns positive work during push-off providing forward propulsion through fluid flowing from the accumulator to the ram. Simulation results are promising: of the total negative work done by the prosthetic ankle over the gait cycle (i.e., the maximum amount of energy available to be stored), $78 \%$ is returned, mainly during push-off; $14 \%$ is carried forward for future gait cycles; and $8 \%$ is lost. The estimated prosthesis height and mass are approximately $26.5 \mathrm{~cm}$ and $2.3 \mathrm{~kg}$. Nonetheless, further work is necessary to realise a prototype for bench and in-vivo testing. By mimicking intact ankle torque and efficiently storing and returning energy at the ankle joint, this new design may contribute to reducing amputees' metabolic cost of walking.
\end{abstract}

Index Terms - Ankle, cam, hydraulics, prosthetics, simulation.

\section{INTRODUCTION}

L OWER-LIMB amputees using prosthetic feet currently $U_{\text {on }}$ the market generally show a metabolic cost of walking higher than anatomically intact subjects, combined with a reduced walking speed. Higher energy expenditures and lower speeds are associated with higher amputation levels [1].

A major reason for these deficits lies in one particular weakness of most prosthetic feet: both conventional and energy storage and return (ESR) feet currently on the market fail to replicate the energy recycling behaviour of an anatomically intact ankle. Specifically, the Achilles tendon stretches during stance prior to push-off and recoils during push-off, producing a large portion of the mechanical work required for forward propulsion and thus reducing the metabolic cost of walking [2], [3]. Conversely, commercially available passive prosthetic feet, including ESR feet with flexible keels, store and return energy

A. Pace (corresponding author) was with the SEE School, University of Salford, Salford, M5 4WT, UK. She is now with the Istituto Italiano di Tecnologia (IIT), Genoa, Italy (e-mail: a.pace1@edu.salford.ac.uk).

J. Gardiner is with the Institute of Life Course and Medical Sciences, University of Liverpool, Liverpool, L7 8TX, UK (e-mail: J.D.Gardiner@liverpool.ac.uk). in an uncontrolled and untimely manner [4]. This occurs because these feet are characterised by a neutral ankle angle, which corresponds to the ankle angle during standing. Therefore, when the foot moves away from neutral, it produces a restoring moment that acts to return it to its neutral position. Therefore, when the foot is plantarflexed, it produces a dorsiflexion moment, rather than the plantarflexion moment seen in normal intact ankle push-off [5]. In other words, the conventional and ESR prosthetic feet currently on the market cannot actively plantarflex beyond their neutral angle, reducing push-off power and leading to an increase in the metabolic cost of walking compared with anatomically intact subjects [6]-[8].

Different researchers have tried to address the limitations of unpowered (passive) prosthetic feet, and thereby mimic the controlled storage and return of mechanical energy seen in anatomically intact subjects. Moreover, it is believed that prosthetic feet that mimic the slope of the anatomically intact ankle joint's torque-versus-angle curve, often referred to as its "quasi-stiffness", improve amputees' gait [9], [10]. However, what is often not mentioned is that this "quasi-stiffness" curve is not the same in the different phases of gait (Fig. 1), implying that a single spring characteristic may not be suitable.

One approach to restoring normal push-off is to power the prosthesis using a battery and electric motor(s). This has the potential to provide both the necessary push-off power and biomimetic ankle torque. A well-known attempt to improve push-off at the end of stance is that of Hugh Herr and colleagues [11]. The resulting commercial device (previously the BiOM, now the Ottobock Empower) relies on electrical power from a battery to produce active push-off. Although there is some elastic energy storage and return, this is similar in nature to that seen in commercial ESR prostheses, with uncontrolled energy return. This critique is supported by the fact that the reduction in metabolic cost for the amputee when using the BiOM, in comparison to using a passive ESR foot, is slightly less than the metabolic equivalent of the energy input from the electric motor, implying that there is no improvement in energy storage and return over commercial ESR prostheses [11]. The disadvantages of powered designs are that batteries are poorly

L. Kenney is with the Health and Society School, University of Salford, Salford, M6 6PU, UK (e-mail: L.P.J.Kenney@ salford.ac.uk).

D. Howard is with the SSE School, University of Salford, Salford, M5 4WT, UK (e-mail: D.Howard@salford.ac.uk). 
suited to the large numbers of charge-discharge cycles that occur in walking, they require charging at regular intervals, and, together with the electric motor(s), they increase the size, weight and cost of the prosthesis [12]. Indeed, in a recently published review of robotic exoskeletons, the electrical power supply problem was highlighted as being “...one (if not the largest) issue..." [13]. For these reasons, the focus of our work has been to improve passive energy storage and return (ESR) so that it provides most, if not all, of the push-off power and, consequently, much smaller batteries are needed (primarily for control, not propulsion).

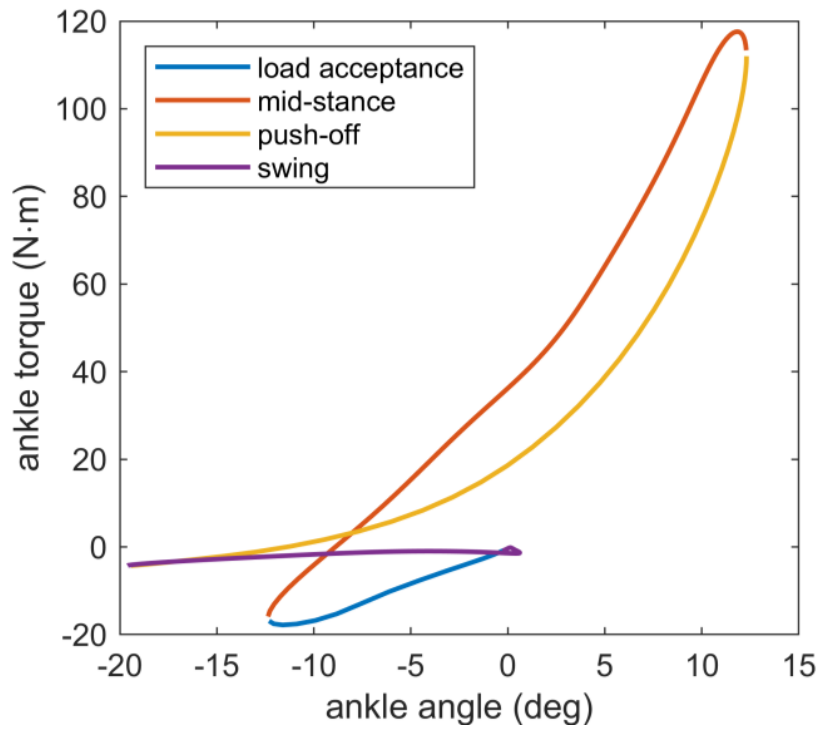

Fig. 1. Torque-versus-angle curve for an anatomically intact ankle joint during level walking. Load acceptance, mid-stance, push-off and swing are displayed with different colours according to the legend. From experimental data collected by Bari [41].

Whether incorporated in powered or unpowered prostheses, advanced ESR concepts for energy storage and return can be broadly classified as either: a) clutch-and-spring devices [6], [11], [14]-[32]; or b) hydraulic devices [33]-[44].

Clutch-and-spring devices have two major disadvantages. Firstly, the control is discrete rather than continuous, locking and releasing the spring, thus preventing smooth biomimetic control. Secondly, such an approach often results in larger volumes and weights, higher complexity [17], and makes interjoint transfer of energy difficult. Energy transfer between joints is of particular importance for higher level amputees who could benefit if the excess of eccentric work at the knee [45], [46] could be stored and used in a controlled manner at other joints. Designs which transfer energy between the knee and ankle joints have used complex and often heavy solutions involving mechanical transmissions. This is the case for passive devices such as [25] and [30], and active prostheses such as the CYBERLEGs Beta-Prosthesis [19]. Indeed, in the next version of this prosthesis - the CYBERLEGs Gamma-Prosthesis - the energy transfer mechanism was removed because of its difficult and unreliable physical implementation [47].

The application of miniature hydraulics is common in prosthetics, mainly for damping and terrain adaptation, such as in the commercially available microprocessor-controlled
Endolite Elan, Ottobock Meridium, Ottobock Triton Smart Ankle, Freedom Kinnex 2.0, and Fillauer Raize. Electricallypowered hydraulic systems have also been developed, including: a knee prosthesis simulator [33]; the SmartLeg transfemoral prosthesis [41]; a semi-active lower-limb prosthesis in which an electric motor-driven hydraulic pump drives knee and ankle hydraulic actuators [42]; and a prosthetic ankle combining electromechanical and hydraulic actuation for damping during load-acceptance, mid-stance groundadaptation, and dorsiflexion for swing foot clearance [43]. Moreover, in the last decades electrohydrostatic actuators have been included in lower-limb prostheses to quickly and smoothly interchange between active and passive tasks, powering pushoff at the ankle and providing swing foot clearance[48], [49].

Although the use of both electrically-powered hydraulic and electrohydrostatic actuators at the ankle can assist with forward propulsion during push-off, delivering biomimetic peak torque and power, this introduces additional weight and volume. Efficiently storing and returning energy over the gait cycle offers a solution by enabling the use of substantially smaller electric motors and batteries, thereby improving amputee mobility by reducing the metabolic cost of walking. As already explained, mechanical systems based on springs are not well suited to producing biomimetic ankle torque or transferring energy between prosthetic joints, and attempts to include electrical energy regeneration in lower-limb prostheses have low efficiency (30-37\%) [12]. We argue that a hydraulic approach, based on using an accumulator to store and return energy, has several potential advantages. Firstly, hydraulic accumulators are well suited for short-term energy storage. For the relatively small amounts of energy stored and returned over one gait cycle, a 50 cc pressurised gas accumulator would be adequate, operating between 190 and 200 bar [50]. Secondly, hydraulic actuation is ideally suited for transferring energy between joints because the transfer mechanism involves only pipes and fluid, rather than gears and linkages, so that excess knee energy can be easily transferred to the ankle. Finally, since hydraulics typically operate at pressures of up to $200 \mathrm{bar}$, hydraulic systems have very high power-densities and are therefore well suited to miniaturisation, an important requirement in prosthetics [44]. For example, during normal walking, the maximum ankle torque is around $100 \mathrm{Nm}$, which would correspond to an actuator that displaces just $5 \mathrm{cc} /$ radian at 200 bar.

Nonetheless, the hydraulic designs described in the literature very rarely include a hydraulic accumulator as an energy store, or hydraulic transfer of energy between prosthetic joints. With the exception of previous work by the authors [44], the few examples that have included an accumulator as an energy store [35], [37], [39] use proportional valves (i.e., variable flow resistances) to control pressures and hence joint torques. This is an inherently dissipative approach that leads to high energy losses. For example, Richter et al. [35] conclude on one such design: "...the system is highly inefficient in an energetic sense...". The approach adopted by Gardiner et al. [44], and also in the work reported here, avoids the energy losses associated 
with pressure control using throttling valves by continuously controlling joint torque through changes in fluid displacement per radian, via respectively a variable displacement actuator (VDA) in [44] and cam driven-rams in this paper.

Based on the arguments above, previous work by the authors focussed on the development of a lower-limb prosthesis using miniature hydraulics. A concept design was developed based on a hydraulic accumulator and a variable displacement actuator (VDA) driven by the ankle joint [44]. This provides continuous biomimetic control of the ankle torque throughout the gait cycle, mimicking the intact ankle, while storing all of the negative work done from heel strike until maximum dorsiflexion, which is then returned in a controlled and timely manner to power push-off. Furthermore, the accumulator could be used as a common energy store allowing the transfer of energy between the knee and ankle joints via pipes. The simulation results were promising and suggested that, despite the significant energy losses involved, a hydraulic VDA-based prosthetic ankle could improve amputee gait by restoring normal push-off. However, for this approach to be a success, a new miniature, low-losses, lightweight VDA would be required that is half the displacement of the smallest commercially available device that could be found. A VDA is a highly specialised and complex component and it would not be appropriate to develop a new VDA just for the prosthetics application. Therefore, in this paper we describe a new concept design, based on simple and readily available miniature hydraulics, which aims to mimic intact ankle torque (see Fig. 1), while storing the negative work done from heel strike to maximum dorsiflexion (A0 and A1 in Fig. 2) in a hydraulic accumulator and returning it during push-off (A2 in Fig. 2). We also present simulation results to illustrate the expected performance of the new design. The novelty in our proposed approach arises from the combination of a hydraulic accumulator for energy storage, the advantages of which are described above, and cam driven rams to achieve biomimetic joint torques while avoiding the use of throttling valves to control pressure.

The new concept design is described in Section II. Section III gives a concise overview of the simulation model, the simulation-based design program, and the simulated performance of the new design. Advantages and limitations of the novel design are discussed in Section IV, and Section V concludes the paper.

\section{The New Design}

A schematic of the concept design for a new hydraulic prosthetic ankle is shown in Fig. 3. Sagittal plane rotation of the prosthetic shank, relative to the prosthetic foot, causes rotation of the ankle shaft and, in turn via the gearbox, rotation of the camshaft. In this way, the ankle joint drives two cams, which in turn drive two hydraulic rams. The "stance cam-ram system" captures the eccentric (negative) work done from foot-flat until maximum dorsiflexion, by pumping oil into the accumulator. The "push-off cam-ram system" returns concentric (positive) work during push-off to provide forward propulsion through fluid flowing from the accumulator to the ram. Directional

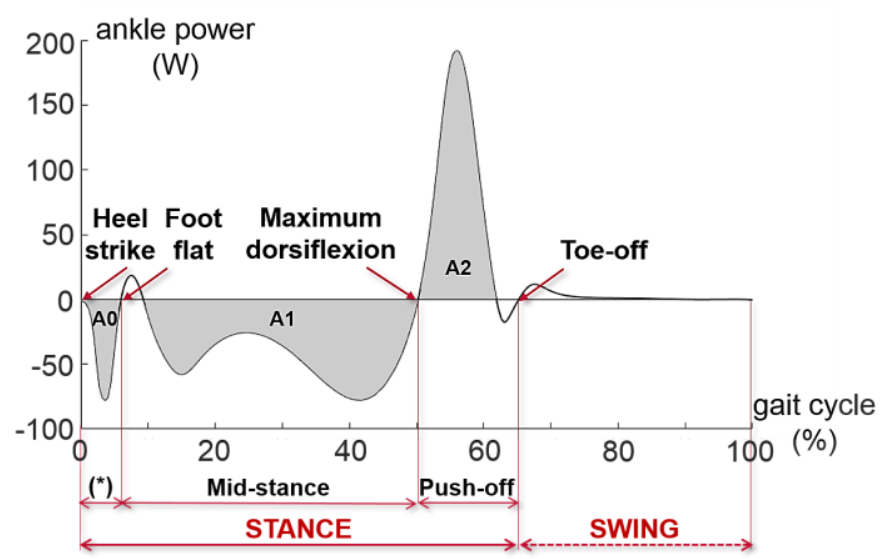

Fig. 2. Ankle power over the full gait cycle in an anatomically intact person walking at self-selected speed (data from Bari [41]). The grey areas delimited by the power curve constitute the ankle energy (work done). The negative areas - A0 and A1 - represent the negative work, that is energy absorption, while the positive area - A 2 - represents the positive work that is energy generation. The symbol (*) represents the "load acceptance" phase of the gait cycle.

control valves (DCVs) connect each hydraulic ram to the tank (i.e., low-pressure accumulator in Fig. 3) during the other ram's working phase, as well as during load acceptance and swing, so that the ram force during these phases is negligible. A torsional spring, which works in parallel to the two cam-ram systems, is an approximate fit to ankle torque during load acceptance (an "ordinary least square" regression fit to the ankle torque data was used to determine the best fit slope and intercept for the spring characteristic), thereby providing good load acceptance, foot ground clearance during swing, and contributing to standing stability.

The cams convert ankle rotation into linear motion of the hydraulic rams and vice versa. The cam profiles are designed to replicate an intact ankle's torque-versus-angle curve over their working phases (i.e., mid-stance for the stance cam-ram system, and push-off for the push-off cam-ram system). Taking into account other sources of torque such as the torsional spring, the stance cam's profile ensures that the ankle torque during midstance (i.e., ankle dorsiflexion) mimics that of an intact ankle. Similarly, the push-off cam's profile ensures that the ankle torque during push-off (i.e., ankle plantarflexion) mimics that of an intact ankle. The gearbox was included to allow cam torque to be less than ankle torque should that be necessary to limit the size of the cam-ram components. However, following a simulation-based design study, we have concluded that the gearbox can be omitted.

Using two cam-rams connected to a single accumulator means it is possible to store and return different amounts of energy during their two working phases. In this way, during level and downhill walking, the energy stored can exceed the energy returned. In other words, the excess eccentric work associated with the torque-versus-angle hysteresis loop can be captured and carried forward to assist with short periods of uphill walking. A $250 c c$ accumulator was chosen because this could be integrated with the prothesis pylon. For illustrative purposes only and assuming it operates between 80 and $100 \mathrm{bar}$, a $250 \mathrm{cc}$ accumulator can store the excess eccentric ankle work of approximately one hundred gait cycles during 


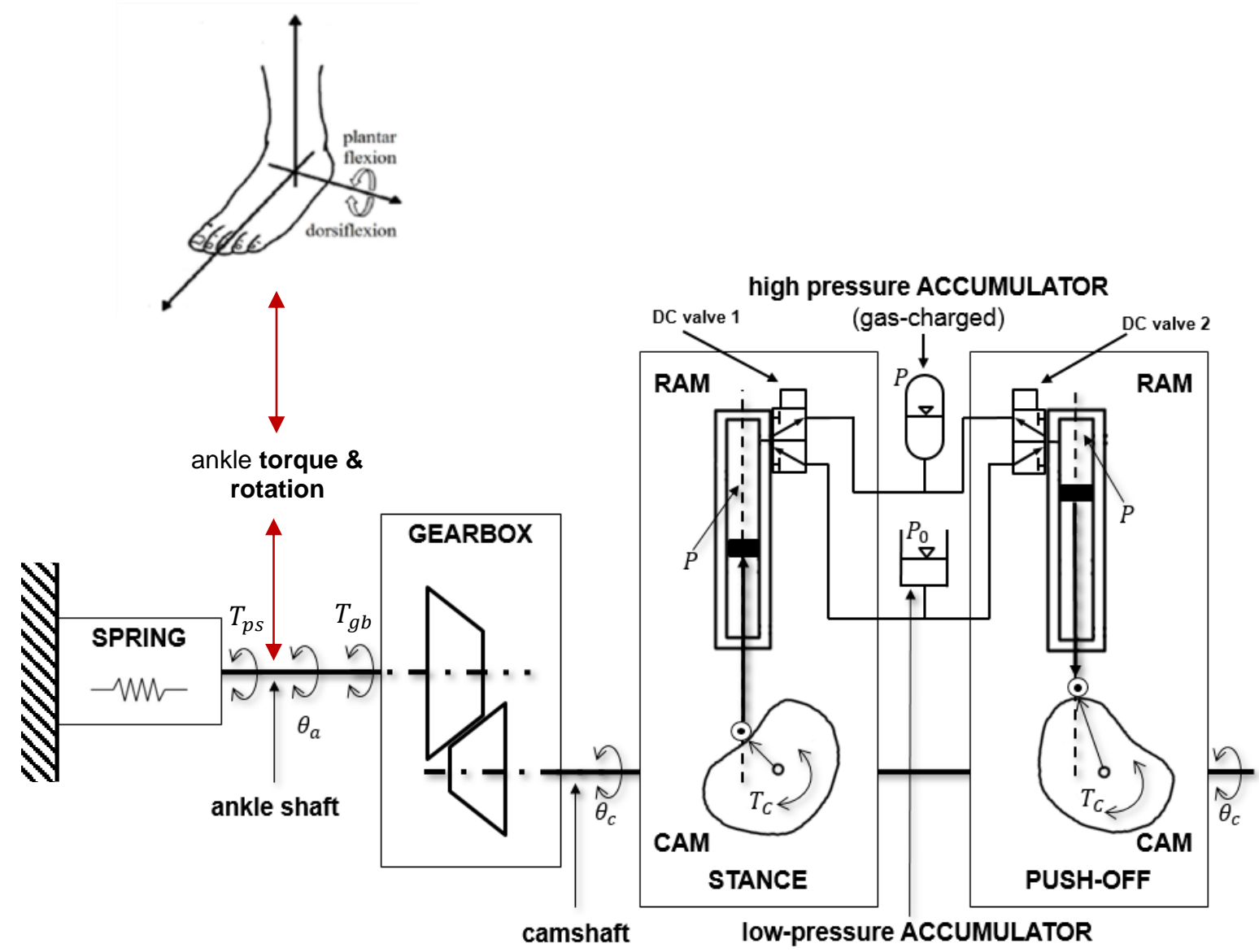

Fig. 3. Schematic of the new concept design, with parallel spring torque $\left(T_{p s}\right)$ and gearbox torque $\left(T_{g b}\right)$ acting on the ankle shaft. The cams, through their rotation $\left(\theta_{c}\right)$, convert ankle rotation $\left(\theta_{a}\right)$ into linear motion of the hydraulic rams and vice versa.

level walking by increasing the pressure from 80 to $100 \mathrm{bar}$ (estimate based on trapezoidal integration assuming an adiabatic process and excess eccentric ankle work of $2.2 \mathrm{~J}$ per gait cycle [51]); and of fifteen gait cycles during downhill walking on a $-15^{\circ}$ slope (based on excess eccentric ankle work of 14.6 J per gait cycle [51]). Even when the accumulator is full (i.e., it has reached its maximum pressure of $100 \mathrm{bar}$ ), the ankle joint will continue to provide the necessary braking during downhill walking by using a pressure relief valve to dissipate eccentric work in the form of heat. During uphill walking, a full accumulator can power approximately ten gait cycles on a $+15^{\circ}$ slope by allowing the pressure to drop from 100 to 80 bar (based on excess concentric ankle work of $21.6 \mathrm{~J}$ [51]). Furthermore, after a review of the literature on accumulators, including technical data sheets (e.g. [52]-[54]), a gas-charged diaphragm accumulator was chosen because they are lighter and better suited to miniaturisation than other types (see also [44]).

Although the work described herein was limited to the design of the prosthetic ankle joint, the use of a single accumulator as a common energy store would allow a multi-joint version of the design to transfer energy between the knee and ankle joints. Indeed, a cam-rams based device at the knee joint, similar to the one described here, could be connected to the same accumulator. In this way, it would be possible to store the eccentric work done at the knee joint and return it at the ankle joint to assist with forward propulsion during push-off.

To estimate the size, particularly the height of the new device, a solid model of the main components affecting height has been created in SolidWorks (v. 2014; Dassault Systemes SolidWorks Corp.). This also serves as an illustration of how the components might be physically assembled. The prosthesis should match the height of the missing anatomy, and weigh no more than the missing anatomy. Considering anthropometric measurements by Winter [55], a $70 \mathrm{~kg}$ subject with a height of $175 \mathrm{~cm}$ [56], average stump length in transtibial amputees according to Isakov et al. [57], and by adding 2 SDs to the mean to include $97.72 \%$ of transtibial amputees, the available height for the new prosthesis assembly, from the ground to the distal connection with the socket, is $28.38 \mathrm{~cm}$. This corresponds to a missing anatomy mass of about $2.43 \mathrm{~kg}$.

Referring to Fig. 4, the new prosthetic ankle is envisioned as a modular component placed between a typical low-profile ESR foot (shown as made of two carbon fibre laminates) and an integrated pylon-accumulator component (not shown). The cams are part of the foot assembly, rotating with it relative to the shank, and the roller-follower-ram assembly is part of the shank. In this way, ankle joint rotation leads to the cams rotating relative to the roller-followers, converting ankle rotation into linear motion of the pistons within the hydraulic rams and, hence, fluid flow between the rams and the accumulator or tank (Fig. 5). The tank would be in the space surrounding the 
hydraulic rams. Components not shown include the parallel spring, the two self-aligning linear ball bearings guiding each follower, the two DCVs, and other hydraulic components. As mentioned above, following a simulation-based design study, we have concluded that the gearbox can be omitted. The connection between the prosthetic ankle and the distal end of the pylon is realised through the male adapter on the top of the ankle unit, with pipes connecting the hydraulic rams in the ankle component to the accumulator in the pylon.

A rough estimate of the height of the whole prosthesis is obtained as follows:

- From the SolidWorks model shown in Fig. 6, the height from the ground to the base of the male adapter pyramid, corresponding to the distal end of the pylon, is approximately $14.5 \mathrm{~cm}$ with the pistons in full outstroke, approximately $3.5 \mathrm{~cm}$ of which is the height of the low-profile ESR foot.

- The 250 cc bespoke accumulator is small enough to be integrated with the prosthesis pylon and is envisioned as a cylinder with an internal diameter of approximately $6 \mathrm{~cm}$ and a height of $10 \mathrm{~cm}$. Thus, the pylon needs to include the accumulator and to allow connection with the ankle unit and the distal end of the socket through the two tubular adapters at its ends. Therefore, a total pylon height of $12 \mathrm{~cm}$ is estimated, from the base of the male adapter pyramid up to the distal end of the socket.

Therefore, the estimated prosthesis height from the ground to the distal end of the socket is approximately $26.5 \mathrm{~cm}$, which is within the available height of $28.38 \mathrm{~cm}$ for the whole assembly. A rough estimate of the mass of the prosthesis was derived, using conservative assumptions (see Table I). Component masses were sourced directly from catalogues for off-the-shelf components, estimated by scaling similar off-the-shelf components, or evaluated directly using Solidworks for bespoke components. The estimated total mass is $2.31 \mathrm{~kg}$, which is comparable with the missing anatomy mass of approximately $2.43 \mathrm{~kg}$.

A more comprehensive description of the design and the simulation-based design process can be found in [50].

\section{SimUlated PERFORMANCE}

A comprehensive mathematical model of the new hydraulic prosthetic ankle was defined [50], including all significant sources of energy loss, in order to obtain a realistic estimate of the performance of the new device and its efficiency.

Based on this mathematical model, a simulation model of the whole system [50]) was implemented in MATLAB (R2018b, The MathWorks, Inc., Natick, MA, USA), which simulates its operation over the whole gait cycle, as it stores and returns energy at the ankle joint. It is driven by the input of ankle angle versus time obtained from experimental gait data (collected by Bari [58]), since this is the only variable the amputee can control once the engineering design of the new device is defined. Conversely, the ankle torque depends on the torques applied by the two cam-ram systems, which in turn depend on the cam profiles - which are fixed - and on the changing pressure in the accumulator. The simulation model calculates the changes in pressure and oil volume in the accumulator, and therefore the energy flows, and the total torque at the ankle joint as outputs.

TABLE I

ESTIMATED MASSES OF THE MAIN COMPONENTS OF THE NEW CONCEPT DESIGN, WITH SOURCES. A MORE DETAILED BREAKDOWN CAN BE FOUND IN [50].

\begin{tabular}{|c|c|c|}
\hline Components & $\begin{array}{c}\text { Estimated } \\
\text { mass (kg) }\end{array}$ & Source \\
\hline $\begin{array}{l}\text { Foot ( } 2 \text { carbon } \\
\text { fiber laminates) }\end{array}$ & 0.11 & SolidWorks estimate \\
\hline Parallel spring & 0.05 & SolidWorks estimate \\
\hline Cams-laminates connector & 0.16 & SolidWorks estimate \\
\hline 2 Cams & 0.07 & SolidWorks estimate \\
\hline $\begin{array}{l}2 \text { Rollers (with } 2 \text { bearings } \\
\text { at each follower) }\end{array}$ & 0.06 & SKF [59], [60] \\
\hline $\begin{array}{l}2 \text { Hydraulic rams (with } \\
\text { return springs \& DCVs) }\end{array}$ & 0.64 & HYDAIRA [61] \\
\hline Aesthetic cover & 0.24 & SolidWorks estimate \\
\hline Male adapter & 0.07 & SolidWorks estimate \\
\hline $\begin{array}{l}\text { Pylon (with the integrated } \\
\text { accumulator) }\end{array}$ & 0.56 & $\begin{array}{l}\text { Estimate based on } \\
\text { [54] and [62] }\end{array}$ \\
\hline Hydraulic oil & 0.15 & $\begin{array}{l}\text { Estimate for an oil } \\
\text { volume of } 175 \mathrm{cc}\end{array}$ \\
\hline Tank & 0.20 & $\begin{array}{c}\text { Estimate based on } \\
\text { [54] and [62] }\end{array}$ \\
\hline Total & 2.31 & \\
\hline
\end{tabular}

A design program, based upon the simulation model and implemented in MATLAB, was used to design the profiles of the two cams to replicate the intact ankle torque, and to specify the two follower return springs that ensure contact between the cams and the rollers [50]. The two cam-ram systems were designed to achieve good performances in their working phases only. Energy losses not directly associated with the two camrams in their working phases were neglected because it was assumed these would have little effect on the selected cam-ram design parameters as the cam-ram's internal forces are much smaller during the non-working phases. Specifically, the MATLAB script converges iteratively to find two cam profiles that enable the system to produce ankle torque curves during the working phases of the two cam-rams that match the required curves, taken from in-vivo experimental data [58]. An iterative solution is necessary because the actual camshaft torque depends upon the dynamics of the two cam-ram systems, which includes velocity and acceleration dependent terms that are determined by the cam profiles. So, using initial estimates of the two cam profiles as inputs, the design program runs the simulation model to calculate the corresponding camshaft torque curves in the two working phases. The error between the calculated (i.e., actual) and required torque curves is then used to update the cam profiles, and this iteration loop is repeated until the error in the two working phases is small. Likewise, an iterative approach was necessary to specify the follower return springs so that cams operate only with compressive normal 


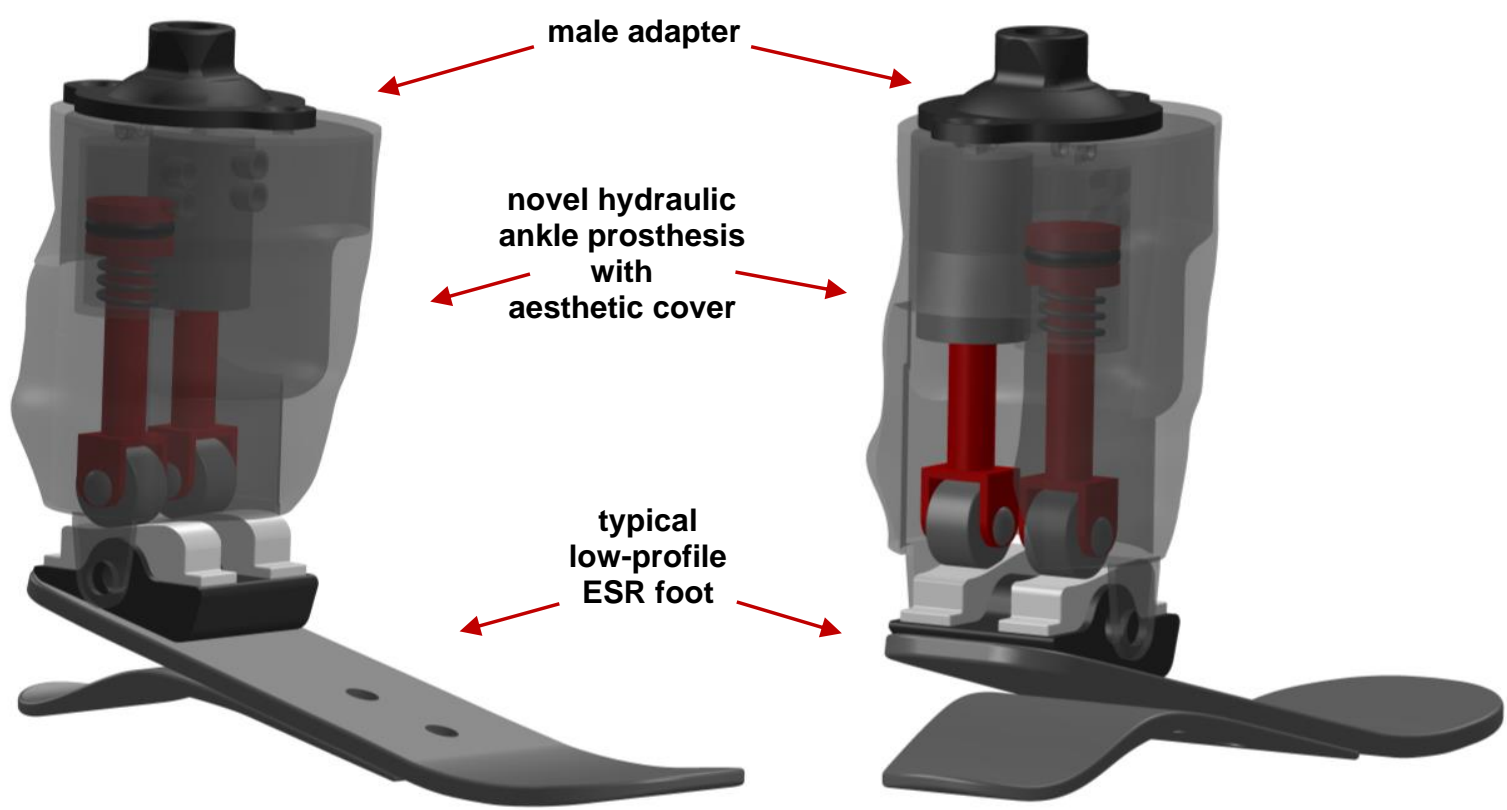

Fig. 4. Solid model of the main components of the novel hydraulic ankle prosthesis (cams, rollers, followers, rams) enclosed in an aesthetic cover and connected to a typical low-profile ESR foot (two carbon fibre laminates). Data for the two cam profiles (the two white components) were exported to SolidWorks from the MATLAB design program and automatically fitted to splines.

forces between cam and roller. Indeed, designing these springs requires knowledge of the cam-roller dynamics, which in turn requires a priori knowledge of the return spring parameters.

Maximum hydraulic pressure, ram bore, and cam-follower configuration were optimised based on the results of multiple
MATLAB simulations to minimise energy losses while achieving realistic overall dimensions [50]. In particular, a maximum hydraulic pressure of $100 \mathrm{bar}$ and a ram bore of $20 \mathrm{~mm}$ allow the two cam-ram systems to achieve the best performances, with lower energy losses and smaller

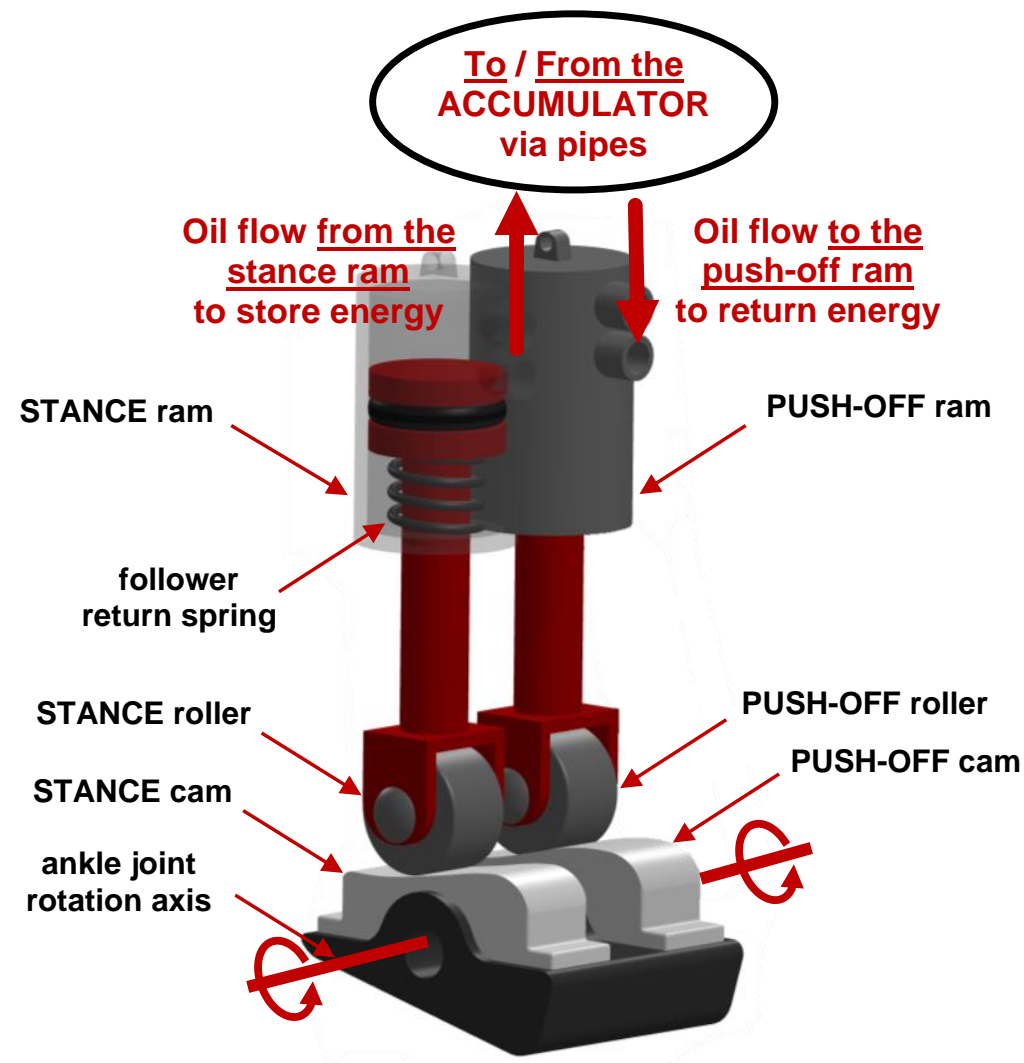

Fig. 5. Detail view of the main components of the novel hydraulic ankle prosthesis: cams, rollers, followers, and hydraulic rams. Note that, although similar, the stance and push-off cams have different profiles. 


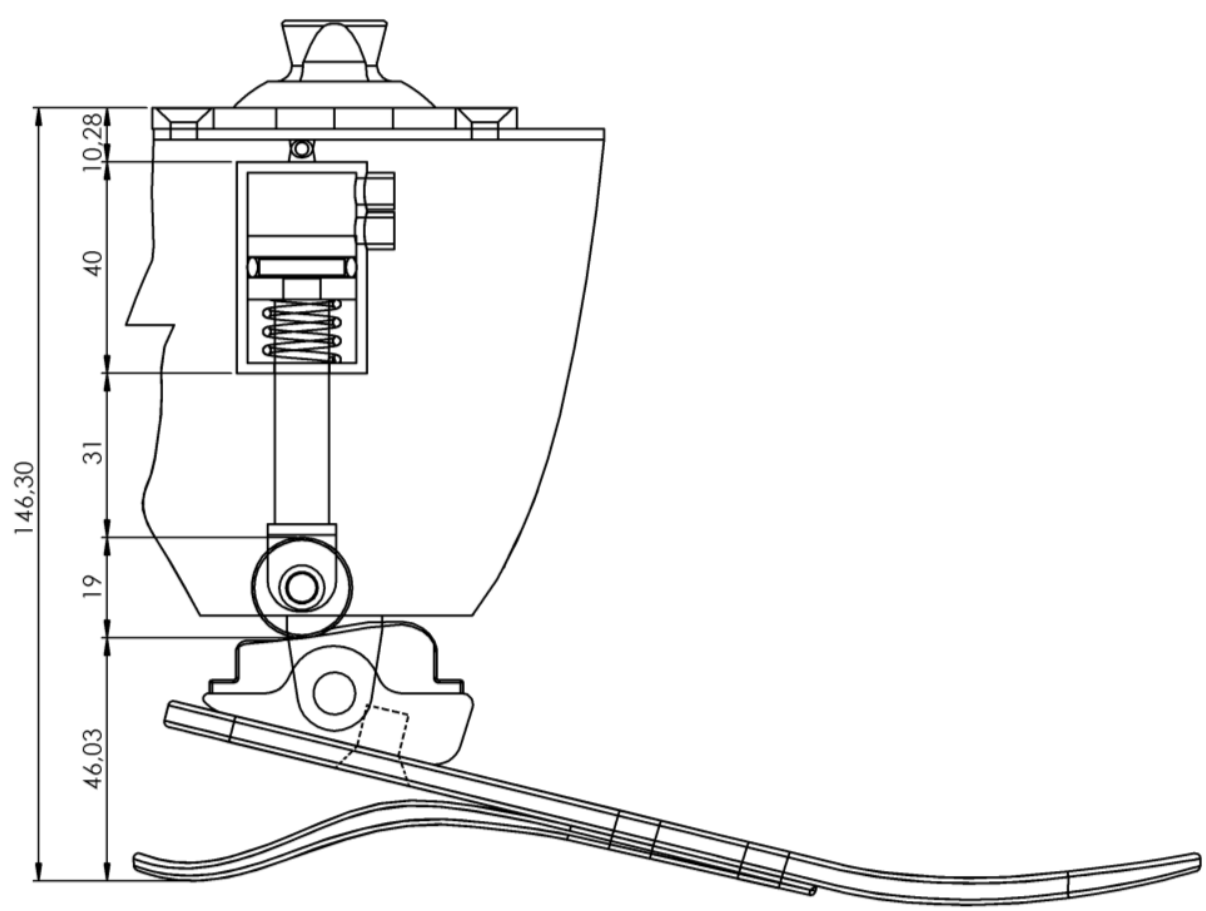

Fig. 6. Lateral view of the prosthetic foot and novel hydraulic ankle (derived from the solid model in Fig. 4). The height of selected components and the total height are shown in $\mathrm{mm}$. Note that, although similar, the stance and push-off cams have different profiles.

dimensions. Furthermore, to withstand the radial cam forces, both cam-ram systems need rollers with minimum diameters of $19 \mathrm{~mm}$, which is the same as the diameter used by Realmuto et al. [63] for the cam-roller-follower system in their active ankle prosthesis.

Fig. 7 shows that, using input ankle angle data for a particular intact ankle [58], the resulting design almost perfectly replicates the torque and power curves of that particular intact ankle during the two working phases (i.e., mid-stance and push-off). Referring to Table II, the predicted ankle torque and power curves were compared with the experimental data, by calculating the root mean square error (RMSE), normalized by the standard deviation (SD) of the experimental data. The normalised RMSEs for the ankle torque and power are less than $4 e-04$ during the two working phases, and less than 0.24 over the whole the gait cycle, confirming that the error is minimal. This differs from simulation and in-vivo test results for unpowered and powered clutch-and-spring devices (for instance [24] and [30]) where torque and power during the working phases are only approximated. These results demonstrate the use of cams and miniature hydraulics to replicate intact ankle power and torque during the key phases of walking. However, because the torsional spring is only an approximate fit to the ankle torque during load acceptance, the match is less good during load acceptance and swing. The disadvantage of using the same torsional spring to bring the ankle back to neutral during swing is that, during push-off plantarflexion, the spring stores more energy than needed to return the foot to neutral during swing. This energy, corresponding to the final peak in power straight after the pushoff peak (marked with an asterisk in Fig. 7 (b)), is lost (dissipated) during swing.
TABLE II

THE RMSE NORMALISED BY SD BETWEEN THE PREDICTED AND THE EXPERIMENTAL ANKLE TORQUE AND POWER DATA, DURING THE DIFFERENT PHASES OF THE GAIT CYCLE

(LOAD ACCEPTANCE (1), MID-STANCE (2), PUSH-OFF (3), AND SWING PHASE (4)) AND FOR THE FULL GAIT CYCLE.

\begin{tabular}{cccccc}
\hline \hline & 1 & 2 & 3 & 4 & $\begin{array}{c}\text { full gait } \\
\text { cycle }\end{array}$ \\
\hline Torque & 0.69 & $2.84 e-04$ & $1.00 e-04$ & 6.55 & 0.09 \\
Power & 0.47 & $3.91 e-04$ & $2.66 e-04$ & 5.03 & 0.24 \\
\hline \hline
\end{tabular}

To identify the most significant sources of energy loss, the following energy losses were modelled: rolling resistance between rollers and cams; friction at the roller and follower bearings, and at the O-rings; flow losses in the pipes and discrete components connecting the two hydraulic rams with the accumulator and tank; heat transfer from the accumulator to the external environment; and compressibility losses in both cylinders and the accumulator.

Fig. 8 shows the energy flows in the new design, over the whole gait cycle, including: the eccentric work done, corresponding to the external energy input at the ankle joint; the concentric work done, mainly during push-off; energy losses; and the net energy stored in the accumulator and carried forward for future gait cycles (e.g., for ascending slopes). These energy terms are displayed as a percentage of the total eccentric work done by the prosthetic ankle over the gait cycle (almost $20.59 \mathrm{~J}$ ), which is the maximum amount of energy available to be stored and returned. The sum of the energy flows leaving the system differs from zero by approximately $-0.83 \%$ of the total eccentric work done, which represents the accuracy of the simulation model, including the numerical approximations 

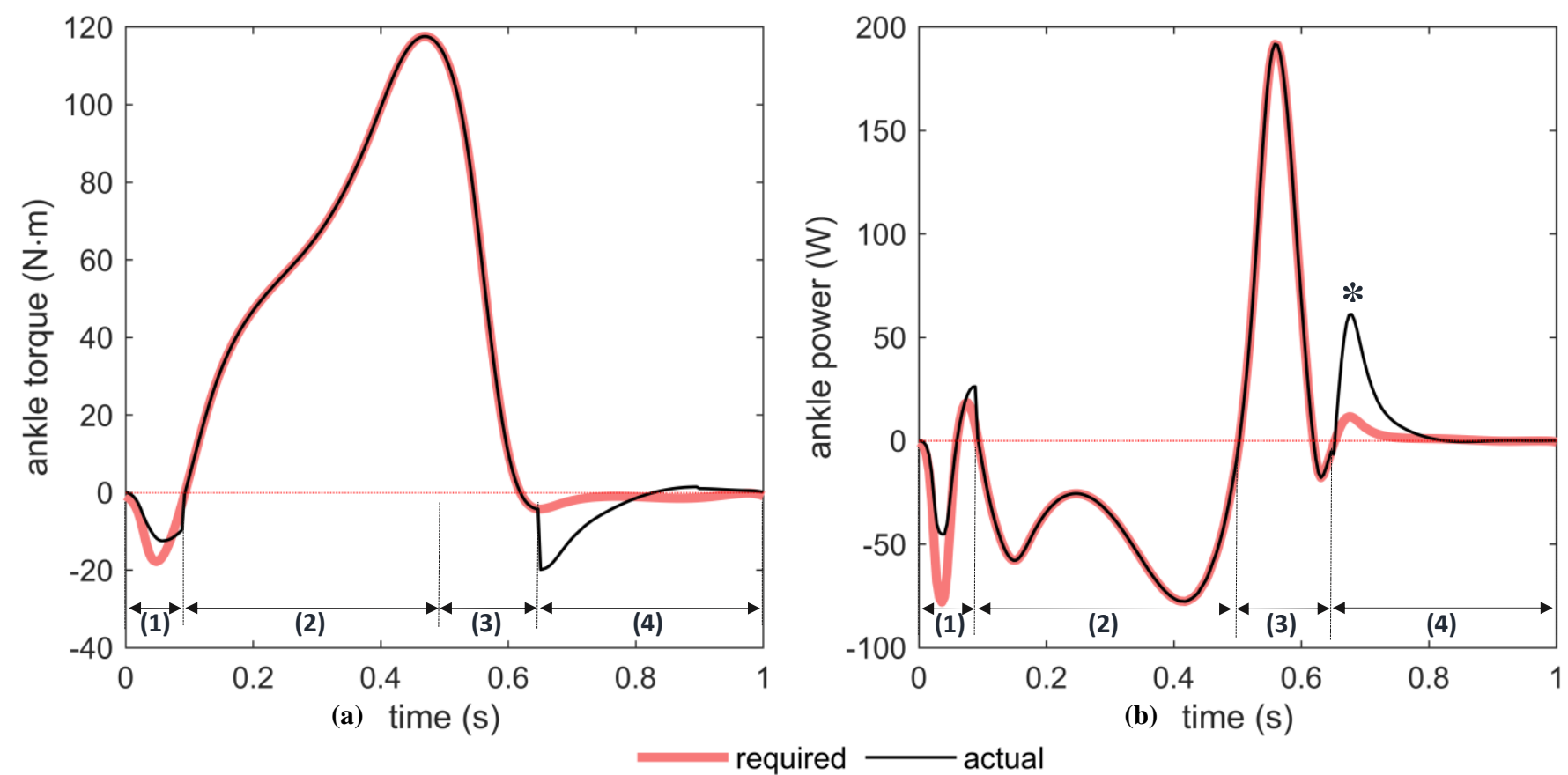

Fig. 7. Torque (a) and power (b) at the prosthetic ankle joint; providing a comparison between the required (light red solid line) - from an anatomically intact subject - and the actual (black solid line) over the gait cycle (load acceptance (1), mid-stance (2), push-off (3), and swing phase (4)). The asterisk above the final peak of the actual ankle power, straight after push-off peak, denotes the energy returned by the torsional spring during swing, which is lost.

inherent in the trapezoidal integration used to calculate these energy terms.

The energy losses over the whole gait cycle are $8.21 \%$ of the total eccentric work done by the prosthetic ankle. The largest sources of energy loss in the final design are O-ring friction in the hydraulic rams $(4.30 \%$ of the total eccentric work), followed by friction in the cam-roller-follower assemblies $(1.67 \%$ in total) and heat loss from the accumulator $(1.60 \%)$. The compressibility losses at valve transitions amount to $0.6 \%$ of the total eccentric work, while flow losses are negligible $(0.05 \%)$. As long as the diameter of the pipe connecting the rams to the accumulator is not reduced below $5 \mathrm{~mm}$, the flow losses are unlikely to exceed $0.2 \%$. A sensitivity study was undertaken to establish where energy losses may be particularly sensitive to changes in the design parameters. The results suggest that, with small changes to the design, it may be possible to reduce the total energy lost over the gait cycle to $5.83 \%$ of the total eccentric work.

A comprehensive description of the mathematical model and the simulation methods can be found in [50].

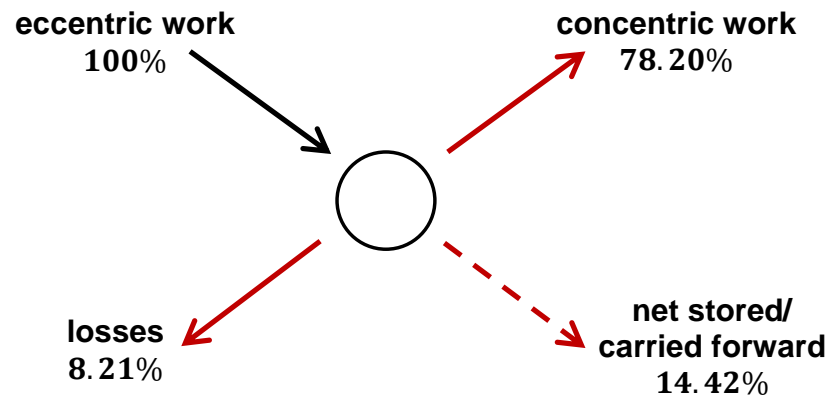

Fig. 8. Energy flows (percentages) in the new hydraulic ankle over the whole gait cycle.

\section{DISCUSSION}

The new design described herein reproduces the ankle torque and power curves seen in anatomically intact gait in a way that that clutch-and-spring designs cannot (e.g., [24] and [30]). Although electrically powered prosthetic ankles can mimic intact ankle torque, they rely largely on battery power to provide forward propulsion during push-off. This requires significant battery capacity, limiting the autonomy of the user due to the need to re-charge at regular intervals. Furthermore, together with the electric motor(s), they increase the size, weight and cost of the prosthesis. The new design offers an alternative approach that has the potential to overcome these disadvantages, exploiting hydraulic energy storage and return to power push-off while providing biomimetic ankle torques. Although the electrical power required by the control system was not considered, this would not be for propulsion and so the battery required would be very small.

A particular advantage of using cams is that it enables biomimetic joint-torque curves to be accurately reproduced (Fig. 7). By using two cam-rams, it is possible to follow different joint-torque versus joint-angle curves during different phases of the gait cycle, which is a feature of normal healthy gait. In other words, hysteresis like curves can be followed. Therefore, it is possible to store and return different amounts of energy during different phases of the gait cycle. In this way, during level and downhill walking, the energy stored can exceed the energy returned, and the excess energy can be carried forward to assist with short periods of uphill walking.

Furthermore, the two cam profiles are specified according to the required ankle torque-versus-angle curve used as input to the design program. This means that cam profiles could be 
unique to the amputee, providing a personalised prosthesis. Indeed, the cams could be manufactured in the prosthetics clinic using a rapid manufacturing technique such as $3 \mathrm{D}$ printing or CNC machining. To achieve this, methods could be developed for basing the required ankle torque-versus-angle curve for first prescription on the amputee's anthropometric, health, fitness and activity characteristics. Conversely, all of the other components would be standardised so that the in-clinic assembly of a personalised prosthetic ankle would be straightforward.

The simulation results presented here show that the new design is far more efficient than, for instance, one of the few examples of orthoses exploiting hydraulics for energy storage purposes: the one developed by Kangude et al. [64]. As shown in Fig. 8, the energy stored in the system over the whole gait cycle $(18.9 \mathrm{~J})$ is approximately $92 \%$ of the total eccentric prosthetic ankle work $(20.59 \mathrm{~J})$. During mid-stance $17.30 \mathrm{~J}$ are stored in the accumulator, of which $14.40 \mathrm{~J}$ are returned during push-off to provide $11.90 \mathrm{~J}$ at the ankle joint. The latter is almost $69 \%$ of the energy stored in the accumulator, as compared with only $7.40 \%$ in Kangude's orthosis because of the high friction losses in the proportional valves.

In addition, hydraulic actuation is ideally suited for transferring energy between joints because the transfer mechanism involves only pipes and fluid, rather than gears and linkages. For example, transfemoral amputees could benefit from energy transfer between the knee and ankle joints, with a single accumulator and cam-ram based joints at the knee and ankle. The use of a single accumulator as a common energy store means that there is no need to explicitly provide for synchronisation between joints. In other words, apart from sharing the accumulator, the two joints can operate independently. In this way, eccentric work at both ankle and knee could be captured, increasing the stored energy carried forward to assist both joints with more demanding tasks, such as climbing stairs or slopes, sit-to-stand movements, etc., although this would need a system that could adapt to different activities and terrains. Future work could investigate the feasibility of a multi-joint system for transfemoral amputees and also of using a similar approach for exoskeletons.

The design could be combined with a battery-powered system to reduce the energy storage and recharge requirements. For example, an electrically driven pump could be used to recharge the accumulator. This could be useful for extended periods of uphill walking. Alternatively, a hand driven pump could be used to recharge the accumulator.

There are a number of limitations to the work reported here. Firstly, the main inputs to the simulation-based design program were the ankle angle and torque over the gait cycle. Experimental averaged data from anatomically intact subjects walking at self-selected speed on level ground, from Bari [58], were used. Although it is within the range of data seen in healthy gait, this input data is optimistic with respect to the amount of eccentric work done. Optimistic data was used because it is believed that amputees could benefit from walking in a manner that provides good push-off if it reduces their metabolic cost of walking. Therefore, the clinical feasibility of the new design will depend on whether the eccentric ankle work needed prior to push-off is justified by the benefits of a more normal push-off, which can only be investigated by in-vivo testing with amputees [44].

Secondly, there are design issues that require further work. As mentioned above, the parallel spring is an approximate fit to the ankle torque during load acceptance only and, therefore, its contributions to swing and standing stability are unlikely to be ideal. In particular, during push-off, the spring stores more energy than needed during early swing and this excess energy is dissipated. Therefore, design changes may need to be considered to reduce this energy loss, which corresponds to the aforementioned final peak in the actual ankle power straight after the push-off peak (marked with an asterisk in Fig. 7 (b)). These changes could increase the stored energy carried forward by $2.6 \mathrm{~J}$ per gait cycle. Furthermore, according to the literature, the quasi-stiffness of the ankle joint during standing is almost three times larger than during the stance phase of gait [65], which is itself considerably higher than that needed for load acceptance. So it may also be necessary to consider design changes to better support standing. In addition, the simulated prosthetic ankle operates with "perfect control", meaning that valve transitions are instantaneous and occur at the ideal moments. Hence, future work should develop and test a control architecture for detecting gait events and driving valve transitions.

Thirdly, the novel hydraulic ankle has been designed for level walking only. Therefore, future work should investigate the feasibility of including real-time adaptation to different slopes, walking speeds, and activities (e.g. stair climbing and sit-to-stand transitions), possibly through the use of 3D cams so that the effective 2D cam could be changed to suit the conditions and activities. Interestingly, the current design would be very suitable for storing the negative work characterising the ankle joint during stand-to-sit [66], which would be stored in the accumulator to power more demanding activities, such as sit-to-stand.

Lastly, this paper describes a simulation study that, while being a valuable tool for exploring the potential of our new design, may not be representative of in-vivo testing outcomes. Simulation results are promising, but further work is necessary to overcome the aforementioned design limitations and manufacture a prototype for bench and in-vivo testing. This will enable us to investigate the performance of this novel device under real conditions and with a variety of amputees with different characteristics (weight, height, etc.).

\section{CONCLUSION}

A novel design for an ESR prosthetic ankle, based on simple miniature hydraulics, has been described. Estimates based on a preliminary SolidWorks model indicate that the height and mass of the device could be acceptable. Matlab simulation results show that the new design is able to mimic intact ankle torque, storing the negative work done from heel strike to maximum dorsiflexion and returning it during push-off in a controlled way. Energy losses are reasonably low and excess stored energy is also available to be carried forward to assist 
with short periods of uphill walking or other demanding tasks.

Thus, by efficiently recycling energy using simple hydraulic components, this novel prosthesis may significantly reduce the metabolic cost of walking of lower-limb amputees. The authors plan to tackle the aforementioned design limitations in future work and then manufacture a prototype for bench and in-vivo testing.

\section{REFERENCES}

[6] S. H. Collins and A. D. Kuo, "Recycling Energy to Restore Impaired Ankle Function during Human Walking," Plos ONE, vol. 5, no. 2, e9307, 17 Feb 2010, doi: 10.1371/journal.pone.0009307.g001.

[7] J. M. Caputo and S. H. Collins, "Prosthetic ankle push-off work reduces metabolic rate but not collision work in non-amputee walking," Sci Rep, vol. 4:7213, 3 Dec 2014, doi: 10.1038/srep07213.

[8] T. P. Huang, K. A. Shorter, P. G. Adamczyk, and A. D. Kuo, "Mechanical and energetic consequences of reduced ankle plantarflexion in human walking," J Exp Biol, vol. 218, no. 22, pp. 35413550, 2015, doi: 10.1242/jeb.113910.

[9] A. H. Hansen, D. S. Childress, S. C. Miff, S. A. Gard, and K. P. Mesplay, "The human ankle during walking: implications for design of biomimetic ankle prostheses," J Biomech, vol. 37, no. 10, pp. 1467-1474, 1 Oct 2004, doi: https://doi.org/10.1016/j.jbiomech.2004.01.017.

[10] R. Versluys, P. Beyl, M. Van Damme, A. Desomer, R. Van Ham, and D. Lefeber, "Prosthetic feet: State-of-the-art review and the importance of mimicking human ankle-foot biomechanics," Disabil Rehabil Assist Technol, vol. 4, no. 2, pp. 65-75, Mar 2009, doi: 10.1080/17483100802715092.

[11] H. Herr and A. M. Grabowski, "Bionic ankle-foot prosthesis normalizes walking gait for persons with leg amputation," in Proc Royal Soc B, 13 Jul 2012, vol. 279, no. 1728, PMC3234569, pp. 457-64, doi: 10.1098/rspb.2011.1194. [Online]. Available: https://www.ncbi.nlm.nih.gov/pubmed/21752817

[12] B. Laschowski, J. McPhee, and J. Andrysek, "Lower-Limb Prostheses and Exoskeletons With Energy Regeneration: Mechatronic Design and Optimization Review," J. Mechanisms Robotics, vol. 11, no. 4, Aug 2019, doi: 10.1115/1.4043460.

[13] A. J. Young and D. P. Ferris, "State of the Art and Future Directions for Lower Limb Robotic Exoskeletons," IEEE Trans Neural Syst Rehabil Eng, vol. 25, no. 2, pp. 171-182, Feb 2017, doi: 10.1109/TNSRE.2016.2521160.

[14] M. E. Carney, "Design and Evaluation of a Reaction-Force Series Elastic Actuator Configurable as Biomimetic Powered Ankle and Knee Prostheses," Ph.D. dissertation, School of Architecture and Planning, MASSACHUSETTS INSTITUTE OF TECHNOLOGY, Cambridge, MA, USA, 2020.

[15] P. Cherelle, G. Mathijssen, Q. Wang, B. Vanderborght, and D. Lefeber, "Advances in Propulsive Bionic Feet and Their Actuation
Principles," Adv Mech Eng, vol. 6, p. 984046, 2014, doi: 10.1155/2014/984046.

[16] P. Cherelle, V. Grosu, M. Cestari, B. Vanderborght, and D. Lefeber, "The AMP-Foot 3, new generation propulsive prosthetic feet with explosive motion characteristics: design and validation," Biomed Eng Online, vol. 15, no. 145, 3, Dec 2016, doi: 10.1186/s12938-0160285-8.

[17] B. Convens et al., "Modeling, Design and Test-Bench Validation of a Semi-Active Propulsive Ankle Prosthesis With a Clutched Series Elastic Actuator," IEEE Robot Autom Lett, vol. 4, no. 2, pp. 18231830, Apr 2019, doi: 10.1109//ra.2019.2897993.

[18] C. Everarts, A. J. C. Thissen, B. Dehez, A. J. Ijspeert, and R. Ronsse, "Control and Design of an Active Prosthesis Based on Adaptive Oscillators," in 10th Belgian Day on Biomedical Engineering - joint meeting with IEEE EMBS Benelux Chapter, Dec 22011.

[19] L. Flynn et al., "The Challenges and Achievements of Experimental Implementation of an Active Transfemoral Prosthesis Based on Biological Quasi-Stiffness: The CYBERLEGs Beta-Prosthesis," Front Neurorobot, vol. 12, p. 80, Dec 4 2018, doi: 10.3389/fnbot.2018.00080.

[20] S. Heins, L. Flynn, H. Laloyaux, J. Geeroms, D. Lefeber, and R. Ronsse, "Compliant Control of a Transfemoral Prosthesis by combining Feed-Forward and Feedback," in 8th IEEE BioRob, New York, USA, Nov - Dec 2020: IEEE, doi: 10.1109/biorob49111.2020.9224434. [Online]. Available: https://dx.doi.org/10.1109/biorob49111.2020.9224434

[21] M. Grimmer et al., "A powered prosthetic ankle joint for walking and running," Biomed Eng Online, vol. 15, no. Suppl 3, p. 141, 19 Dec 2016, doi: 10.1186/s12938-016-0286-7.

[22] J. K. Hitt, R. Bellman, M. Holgate, T. G. Sugar, and K. W. Hollander, "The SPARKy (Spring Ankle With Regenerative Kinetics) Project: Design and Analysis of a Robotic Transtibial Prosthesis With Regenerative Kinetics," in ASME 2007 International Design Engineering Technical Conferences and Computers and Information in Engineering Conference, 2007, vol. 5: 6th International Conference on Multibody Systems, Nonlinear Dynamics, and Control, Parts A, B, and C, pp. 1587-1596, doi: 10.1115/detc2007-34512. [Online]. Available: https://doi.org/10.1115/DETC2007-34512

[23] M. Holgate and T. G. Sugar, "Active Compliant Parallel Mechanisms," in ASME 2014 International Design Engineering Technical Conferences and Computers and Information in Engineering Conference, Buffalo, New York, USA, 17-20 Aug 2014, vol. 5A: 38th Mechanisms and Robotics Conference, V05AT08A080, doi: 10.1115/detc2014-35668. [Online]. Available: https://doi.org/10.1115/DETC2014-35668

[24] B. E. Lawson, J. Mitchell, D. Truex, A. Shultz, E. Ledoux, and M. Goldfarb, "A Robotic Leg Prosthesis: Design, Control, and Implementation," IEEE Robot Autom Mag, vol. 21, no. 4, pp. 70-81, 2014, doi: 10.1109/MRA.2014.2360303.

[25] A. Matthys, P. Cherelle, M. Van Damme, B. Vanderborght, and D. Lefeber, "Concept and design of the HEKTA (Harvest Energy from the Knee and Transfer it to the Ankle) transfemoral prosthesis," presented at the IEEE BioRob, Rome, Italy, 24-27 Jun, 2012.

[26] A. H. Shultz, J. E. Mitchell, D. Truex, B. E. Lawson, and M. Goldfarb, "Preliminary evaluation of a walking controller for a powered ankle prosthesis," in 2013 IEEE Int Conf Robot Autom, 610 May 2013, pp. 4838-4843, doi: 10.1109/ICRA.2013.6631267.

[27] A. H. Shultz, B. E. Lawson, and M. Goldfarb, "Variable Cadence Walking and Ground Adaptive Standing With a Powered Ankle Prosthesis," IEEE Trans. Neural Syst. Rehabilitation Eng., vol. 24, no. 4, pp. 495-505, Apr 2016, doi: 10.1109/tnsre.2015.2428196.

[28] S. Culver, H. Bartlett, A. H. Shultz, and M. Goldfarb, "A Stair Ascent and Descent Controller for a Powered Ankle Prosthesis," IEEE Trans. Neural Syst. Rehabilitation Eng., vol. 26, no. 5, pp. 993-1002, May 2018, doi: 10.1109/tnsre.2018.2819508.

[29] F. Sup, H. A. Varol, J. Mitchell, T. J. Withrow, and M. Goldfarb, "Preliminary Evaluations of a Self-Contained Anthropomorphic Transfemoral Prosthesis," IEEE/ASME Trans Mechatronics, vol. 14, no. 6, pp. 667-676, 2009, doi: 10.1109/tmech.2009.2032688. R. Unal et al., "Modeling of WalkMECH: a fully-passive energyefficient transfemoral prosthesis prototype," in 2013 IEEE Int Conf Rehabil Robot, 24-26 Jun 2013, 2013/11/05 ed., p. 6650406, doi: 10.1109/icorr.2013.6650406. 
[31] R. J. Williams, A. H. Hansen, and S. A. Gard, "Prosthetic AnkleFoot Mechanism Capable of Automatic Adaptation to the Walking Surface," J Biomech Eng, vol. 131, no. 3, p. 035002, 2009, doi: 10.1115/1.3005335.

[32] H. She, J. Zhu, Y. Tian, Y. Wang, and Q. Huang, "Design of a powered ankle-foot prosthesis with an adjustable stiffness toe joint," Adv. Robot., vol. 34, no. 10, pp. 689-697, 2020/05/18 2020, doi: $10.1080 / 01691864.2020 .1750479$.

[33] W. C. Flowers and R. W. Mann, "An Electrohydraulic Knee-Torque Controller for a Prosthesis Simulator," J Biomech Eng, vol. 99, no. 1, pp. 3-8, 1977, doi: 10.1115/1.3426266.

[34] K. Koganezawa, H. Fujimoto, and I. Kato, "Multifunctional aboveknee prosthesis for stairs' walking," Prosthet Orthot Int, vol. 11, no. 3, pp. 139-45, Dec 1987, doi: 10.3109/03093648709078198.

[35] H. Richter, X. Hui, A. van den Bogert, and D. Simon, "Semiactive virtual control of a hydraulic prosthetic knee," in IEEE CCA, 19-22 Sept 2016, pp. 422-429, doi: 10.1109/CCA.2016.7587867.

[36] S. Sophyn and K. Koganezawa, "ABOVE KNEE PROSTHESIS FOR ASCENDING/DESCENDING STAIRS WITH NO EXTERNAL ENERGY SOURCE," in Assistive Robotics Proceedings of the 18th International Conference on CLAWAR 2015, H. Su, T. Wang, M. O. Tokhi, and G. S. Virk, Eds., 2015: World Scientific Publishing Co. Pte. Ltd. [Online]. Available: https://books.google.co.uk/books?id=C3efCgAAQBAJ\&pg=PA35 \&lpg=PA35\&dq=2015+Above+knee+prosthesis+forascending $/ \mathrm{des}$ cending+stairs+with+no+external+energy+source\&source=bl\&ots $=10 \mathrm{xsPKdpsi} \&$ sig=ACfU3U2CgTfe-

YbyIJw9vCQ2MSRjIaIvDg\&hl=it\&sa $=X \& v e d=2 a h U K E w j X y-$ Cq4NDIAhX9TxUIHd_pBRAQ6AEwBHoECAkQAQ\#v=onepag e\&q\&f=true.

[37] A. J. van den Bogert, S. Samorezov, B. L. Davis, and W. A. Smith, "Modeling and Optimal Control of an Energy-Storing Prosthetic Knee," J Biomech Eng, vol. 134, pp. 051007-1/8, May 2012, doi: 10.1115/1.4006680.

[38] A. B. Wilson, "Recent Advances in Above-Knee Prosthetics," Artif Limbs, vol. 12, no. 2, pp. 1-27, 1968. [Online]. Available: http://www.oandplibrary.com/al/pdf/1968_02 001.pdf.

[39] T. Wilmot et al., "Biogeography-Based Optimization for Hydraulic Prosthetic Knee Control," in Medical CPS Workshop, Philadelphia, Pennsylvania, Apr 2013, pp. 18-25.

[40] F. Gao, Y. Liu, and W.-H. Liao, "Design of Powered Ankle-Foot Prosthesis With Nonlinear Parallel Spring Mechanism," J. Mech. Des., vol. 140, no. 5, 2018, doi: 10.1115/1.4039385.

[41] R. Dedić and H. Dindo, "SmartLeg: An intelligent active robotic prosthesis for lower-limb amputees," in IEEE ICAT, Oct 27-29 2011, pp. 1-7, doi: 10.1109/ICAT.2011.6102090.

[42] M. V. Pillai, H. Kazerooni, and A. Hurwich, "Design of a semiactive knee-ankle prosthesis," in IEEE ICRA, Shanghai, China, May 9-13 2011, doi: 10.1109/icra.2011.5980178. [Online]. Available: https://dx.doi.org/10.1109/icra.2011.5980178

[43] H. L. Bartlett, S. T. King, M. Goldfarb, and B. E. Lawson, "A SemiPowered Ankle Prosthesis and Unified Controller for Level and Sloped Walking," IEEE Trans. Neural Syst. Rehabilitation Eng., pp. 1-1, 2021, doi: 10.1109/tnsre.2021.3049194.

[44] J. Gardiner et al., "Performance of optimised prosthetic ankle designs that are based on a hydraulic variable displacement actuator (VDA)," IEEE Trans. Neural Syst. Rehabilitation Eng., vol. 25, no. 12, pp. $2418-2426,2$ Nov 2017, doi: 10.1109/TNSRE.2017.2763999.

[45] A. E. Ferris, J. M. Aldridge, C. A. Rabago, and J. M. Wilken, "Evaluation of a powered ankle-foot prosthetic system during walking," Arch Phys Med Rehabil, vol. 93, no. 11, pp. 1911-8, Nov 2012, doi: 10.1016/j.apmr.2012.06.009.

[46] A. D. Segal et al., "Kinematic and kinetic comparisons of transfemoral amputee gait using C-Leg and Mauch SNS prosthetic knees," J Rehabil Res Dev, vol. 43, no. 7, pp. 857-70, Nov-Dec 2006, doi: 10.1682/jrrd.2005.09.0147.

[47] L. Flynn, J. Geeroms, S. Heins, B. Vanderborght, and D. Lefeber, "Estimation of Energy Minimizing Series Elastic Spring Stiffness for an Active Knee Prosthesis," in IEEE BioRob, New York, USA, Nov - Dec 2020: IEEE, doi: 10.1109/biorob49111.2020.9224393. [Online]. https://dx.doi.org/10.1109/biorob49111.2020.9224393

[48] T. Yu, A. R. Plummer, P. Iravani, J. Bhatti, S. Zahedi, and D. Moser, "The Design, Control, and Testing of an Integrated
Electrohydrostatic Powered Ankle Prosthesis," IEEE ASME Trans. Mechatron., vol. 24, no. 3, pp. 1011-1022, Jun 2019, doi: 10.1109/tmech.2019.2911685.

[49] J. Fang, X. Wang, R. Li, S. Wang, and W. Wang, "Active Ankle Prosthesis Powered by Electrohydrostatic Actuation Technology: Design and Implementation," in CSAA/IET AUS, Guiyan, China, Jun 2018: Institution of Engineering and Technology, doi: 10.1049/cp.2018.0177. [Online]. Available: https://dx.doi.org/10.1049/cp.2018.0177

[50] A. Pace, "A novel hydraulic energy-storage-and-return prosthetic ankle: design, modelling and simulation," Ph.D. dissertation, School of Science, Engineering \& Environment, University of Salford, Salford, UK, 2020. [Online]. Available: http://usir.salford.ac.uk/id/eprint/58264/

[51] A. N. Lay, C. J. Hass, and R. J. Gregor, "The effects of sloped surfaces on locomotion: A kinematic and kinetic analysis," $J$ Biomech, vol. 39, no. 9, pp. 1621-1628, 1 Jan 2006, doi: https://doi.org/10.1016/j.jbiomech.2005.05.005.

[52] HYDAC TECHNOLOGY CORPORATION. Accumulators: Bladder, Piston, Diaphragm, PN\#02068195/ 1.15/ACU1102-1326, $2015 . \quad$ [Online]. Available: https://autodocbox.com/Hybrid/71938690-Pn-1-15-acuaccumulators-bladder-piston-diaphragm.html. Accessed: May 19, 2021.

[53] REXROTH. Diaphragm-type accumulator, RE 50150/01.2013, Replaces: 11/07, 2013. [Online]. Available: https://www.duncanrogers.com/literature/re50150_2013-01.pdf. Accessed: May 19, 2021.

[54] Parker Hannifin. Accumulator, Accessories and Spares Catalogue, MSG10-4004/UK rev. 10, 2018. [Online]. Available: https://www.parker.com/Literature/Accumulator\%20\&\%20Cooler \%20Division\%20-\%20Europe/Accumulator-Catalogue.pdf.

Accessed: May 19, 2021.

[55] D. A. Winter, Biomechanics and Motor Control of Human Movement, 4th ed. Hoboken, NJ, USA: John Wiley \& Sons, Inc., 2009.

[56] D. A. Winter, "Kinematic and kinetic patterns in human gait: Variability and compensating effects," Hum Movement Sci, vol. 3, no. 1, pp. 51-76, 1 Mar 1984, doi: https://doi.org/10.1016/01679457(84)90005-8.

[57] E. Isakov, H. Burger, M. Gregoric, and C. Marincek, "Stump length as related to atrophy and strength of the thigh muscles in trans-tibial amputees," Prosthet Orthot Int, vol. 20, no. 2, pp. 96-100, 1996.

[58] A. Z. Bari, "An efficient energy storage and return prosthesis," Ph.D. dissertation, Centre for Health Sciences Research, University of Salford, Salford, UK, 2013.

[59] SKF. Rolling bearings, PUB BU/P1 10000/2 EN, 2013. [Online]. Available: http://www.skf.com/binary/77-121486/SKF-rollingbearings-catalogue.pdf.

[60] SKF. Linear bearings and units with SKF factory pre-lubrication, PUB PT/P1 4182/2 EN, 2014. [Online]. Available: https://www.motion-

products.ru/upload/iblock/a65/a65dc3a557a9514d4c391083cebf1a cb.pdf.

[61] HYDAIRA. Hydraulic Cylinders. [Online]. Available: https://specken-

drumag.com/uploads/pdfs/KAT_Hydraulikzylinder_en.pdf.

[62] Crompton Technology Group Ltd. http://www.ctgltd.com/ (accessed May 19, 2021.

[63] J. Realmuto, G. Klute, and S. Devasia, "Nonlinear Passive CamBased Springs for Powered Ankle Prostheses," J. Med. Device., vol. 9, no. 1, 2015, doi: 10.1115/1.4028653.

[64] A. Kangude, B. Burgstahler, J. Kakastys, and W. Durfee, "Single channel hybrid FES gait system using an energy storing orthosis: preliminary design," in IEEE EMBS, Dec 8 2009, pp. 6798-801, doi: 10.1109/iembs.2009.5333976.

[65] M. K. Shepherd and E. J. Rouse, "Design of a quasi-passive anklefoot prosthesis with biomimetic, variable stiffness," in IEEE ICRA, May 29 - Jun 3 2017, pp. 6672-6678, doi: 10.1109/ICRA.2017.7989788.

[66] B. Laschowski, R. S. Razavian, and J. McPhee, "Simulation of Stand-to-Sit Biomechanics for Robotic Exoskeletons and Prostheses with Energy Regeneration," Simulation of Stand-to-Sit Biomechanics for Robotic Exoskeletons and Prostheses with Energy Regeneration, pp. 1-1, 2021, doi: 10.1109/tmrb.2021.3058323. 